PHYSICAL REVIEW D 95, 069902(E) (2017)

\title{
Erratum: Big brake singularity is accommodated as an exotic quintessence field \\ [Phys. Rev. D 93, 043524 (2016)]
}

Luis P. Chimento and Martín G. Richarte

(Received 2 March 2017; published 16 March 2017)

DOI: 10.1103/PhysRevD.95.069902

There is a typo in Eq. (63), namely, the last term should be multiplied by gravitational potential $\psi$. Therefore the equation can be recast as

$$
\ddot{\psi}+3 \mathcal{H}\left(1+3 c_{a}^{2}\right) \dot{\psi}+\left\{2 \dot{\mathcal{H}}+k^{2} c_{s \mathrm{rf}}^{2}+\left(3 c_{a}^{2}+1\right) \mathcal{H}^{2}\right\} \psi=0 .
$$

This typo does not affect the results presented in the article and all the conclusions remain unchanged. 